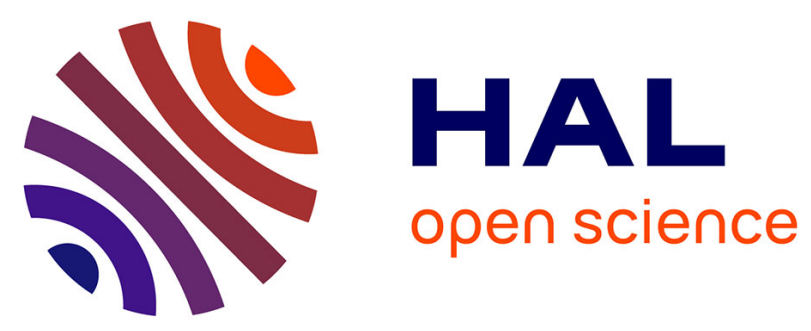

\title{
Dielectric relaxations in amorphous polymers with complex chain architectures
}

Eric Dantras, Emeline Dudognon, Valérie Samouillan, Jérôme Menegotto, Alain Bernès, Philippe Demont, Colette Lacabanne

\section{- To cite this version:}

Eric Dantras, Emeline Dudognon, Valérie Samouillan, Jérôme Menegotto, Alain Bernès, et al.. Dielectric relaxations in amorphous polymers with complex chain architectures. Journal of Non-Crystalline Solids, 2002, vol. 307-310, pp. 671-678. 10.1016/S0022-3093(02)01530-2 . hal-01308048

\section{HAL Id: hal-01308048 \\ https://hal.science/hal-01308048}

Submitted on 27 Apr 2016

HAL is a multi-disciplinary open access archive for the deposit and dissemination of scientific research documents, whether they are published or not. The documents may come from teaching and research institutions in France or abroad, or from public or private research centers.
L'archive ouverte pluridisciplinaire HAL, est destinée au dépôt et à la diffusion de documents scientifiques de niveau recherche, publiés ou non, émanant des établissements d'enseignement et de recherche français ou étrangers, des laboratoires publics ou privés. 


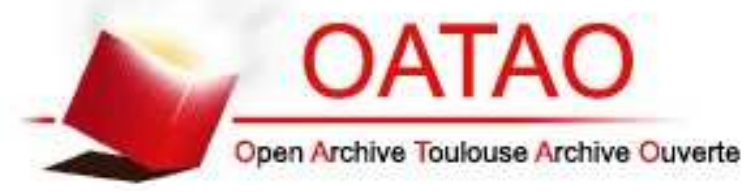

\section{Open Archive TOULOUSE Archive Ouverte (OATAO)}

OATAO is an open access repository that collects the work of Toulouse researchers and makes it freely available over the web where possible.

This is an author-deposited version published in : http://oatao.univ-toulouse.fr/ Eprints ID : 14312

To link to this article : DOI : 10.1016/S0022-3093(02)01530-2

URL : http://dx.doi.org/10.1016/S0022-3093(02)01530-2

To cite this version : Dantras, Eric and Dudognon, Emeline and Samouillan, Valérie and Menegotto, Jérôme and Bernès, Alain and Demont, Philippe and Lacabanne, Colette Dielectric relaxations in amorphous polymers with complex chain architectures. (2002) Journal of Non-Crystalline Solids, vol. 307-310. pp. 671-678. ISSN 0022-3093

Any correspondance concerning this service should be sent to the repository administrator: staff-oatao@ listes-diff.inp-toulouse.fr 


\title{
Dielectric relaxations in amorphous polymers with complex chain architectures
}

\author{
E. Dantras *, E. Dudognon, V. Samouillan, J. Menegotto, A. Bernès, \\ P. Demont, C. Lacabanne \\ Laboratoire de Physique des Polymères, Université Paul Sabatier, 118 route de Narbonne, 31062 Toulouse cedex 4, France
}

\begin{abstract}
By combining thermostimulated current and dynamic dielectric spectroscopies, the dielectric properties characteristic of the molecular mobility can be described over a very broad frequency range. The study of dielectric relaxations of phosphorus-containing dendrimers in the sub-glass transition region, and of poly( $n$-alkyl methacrylates) at and above the glass transition region allows us to gain insight into the molecular dynamics of complex architecture systems. From these sets of data we make an attempt to propose a structural interpretation of molecular mobility through the glass transition.
\end{abstract}

\section{Introduction}

The aim of this work is to improve the knowledge of the dielectric behavior of amorphous polymers with complex chain architecture by using a combination of dynamic dielectric spectroscopy (DDS) and thermostimulated currents (TSC). We significantly broaden the accessible frequency range $\left(10^{6}-10^{-4} \mathrm{~Hz}\right)$ and then we can follow with good accuracy the shift of the various relaxation modes as a function of temperature and frequency. Moreover, the decomposition of complex spectra in elementary relaxation processes can be performed experimentally by TSC. The temperature dependence of elementary relaxation times is analyzed,

\footnotetext{
* Corresponding author.

E-mail address: dantras@cict.fr (E. Dantras).
}

which allows the study of spatially distributed molecular dynamics of complex materials. In the sub-glass transition region, secondary relaxation modes $(\beta)$, generally due to localized processes, have been considered. The example of phosphoruscontaining dendrimers of various generations i.e. with different complexity, will be given. In the glass transition region, the main relaxation of complex amorphous macromolecules is described through the study of biopolymers like elastin. And finally, the series of poly( $n$-alkyl methacrylates) has been chosen as model amorphous linear polymers to investigate complex relaxation processes in the glass transition and upper regions.

\section{Experimental section}

Broadband dielectric measurements were performed using a Novocontrol BDS 4000 covering a 
frequency range of $10^{-2} / 10^{6} \mathrm{~Hz}$ with 10 points per decade. Experiments were carried out within a temperature range $-150 / 130{ }^{\circ} \mathrm{C}$. Dielectric isotherms were measured every $5{ }^{\circ} \mathrm{C}$. Before each frequency scan, temperature was kept constant to within $\pm 0.2^{\circ} \mathrm{C}$. The real $\varepsilon_{\mathrm{T}}^{\prime}$ and imaginary $\varepsilon_{\mathrm{T}}^{\prime \prime}$ parts of the relative complex permittivity $\varepsilon_{\mathrm{T}}^{*}$, were measured as a function of the frequency $F$ at a given temperature $T$ and fitted by Havriliak-Negami (HN) function

$\varepsilon_{\mathrm{T}}^{*}(\omega)=\varepsilon_{\infty}+\Delta \varepsilon /\left(1+\left(\mathrm{i} \omega \tau_{\mathrm{HN}}\right)^{\alpha_{\mathrm{HN}}}\right)^{\beta_{\mathrm{HN}}}$,

where $\varepsilon_{\infty}$ is the real permittivity for high frequencies, $\Delta \varepsilon$ is the relaxation strength and $\alpha_{\mathrm{HN}}$ and $\beta_{\mathrm{HN}}$ are the HN parameters. For TSC experiments, the sample was polarized by an electrostatic field $E_{\mathrm{P}}=400 \mathrm{kV} / \mathrm{m}$ during $t_{\mathrm{p}}=2$ min over a temperature range from the polarization temperature $T_{\mathrm{P}}=-100{ }^{\circ} \mathrm{C}$ down to the freezing temperature $T_{0}=\mathrm{LNT}$. Then, the field was turned off and the depolarization current was recorded with a constant heating rate $\left(q_{\mathrm{h}}=+7{ }^{\circ} \mathrm{C} / \mathrm{min}\right)$, so that the equivalent frequency of the TSC spectra was $f_{\text {eq }} \sim$ $10^{-3}-10^{-4} \mathrm{~Hz}$. TSC allows us to follow the shift of complex relaxation modes on a frequency range complementary from the frequency window accessible by DDS. The fractional polarization (FP) method permits us to describe the fine structure of these modes. For windowing experiments, the $i$ th elementary peak was obtained by polarizing a sample for $t_{\mathrm{p}}=2 \mathrm{~min}$ at $T_{\mathrm{p}}^{i}$. Then the sample was short-circuited at $T_{\mathrm{d}}^{i}=T_{\mathrm{p}}^{i}-\Delta T_{\mathrm{p}}$, and the temperature was maintained constant for $t_{\mathrm{d}}=t_{\mathrm{p}}$. Then the sample was cooled at $T_{0}^{i}=T_{\mathrm{p}}^{i}-50{ }^{\circ} \mathrm{C}$, and the depolarization current was recorded in the same way as for the complex mode. The temperature dependence of relaxation time $\tau(T)$ associated with each elementary spectrum can be determined from the Bucci-Fieschi's formalism [1].

\section{Results}

\subsection{Sub-glass transition region: secondary relax- ation in dendrimers}

Evolution of dendrimers generation allows us to follow relaxational complexity and molecular structure relationships. Phosphorus-containing dendrimers are characterized by a regularly branched tree-like architecture (self-similar or fractal).

From a phosphorus core constituted by a strong dipole, these dendrimers are synthesized by a divergent process that consists on iteration of chemical steps. On the same core, radially branched layers are attached. Dendrimers are synthesized generation after generation, step by step. The state of the art is reached with this phosphorus containing dendrimers series: the 12th generation; but details of synthesis of this amorphous polymers are published elsewhere [2,3]. Numbering of these compounds hereafter will be done as follows: $\left[G_{g}^{\prime}\right]$ in which $g$ is the number of generation - here $g=0,1$ and $2-G^{\prime}$ corresponds to a dendrimer with terminal aldehyde groups and a $\mathrm{P}=\mathrm{S}$ core. Phosphorus-containing dendrimers of generation 0 , with a $\mathrm{P}=\mathrm{O}$ core will be designated as $\left[G_{0}^{\prime}\right.$ $(\mathrm{P}=\mathrm{O})$ ] for chemical species with an aldehyde end group, and $\left[G_{0}(\mathrm{P}=\mathrm{O})\right]$ for chemical species without aldehyde end group. For $\left[G_{0}^{\prime}\right]$, the sample exhibiting no glass transition, no $\alpha$ dielectric relaxation is observed. But dielectric loss profile (DDS results) confirms previous data on different dendritic macromolecules [4]: a quasi-Debye mode $\beta_{0}^{\left[G^{\prime}\right]}$ (Fig. 1), confirmed by HN parameters is pointed at low frequency and low temperature.

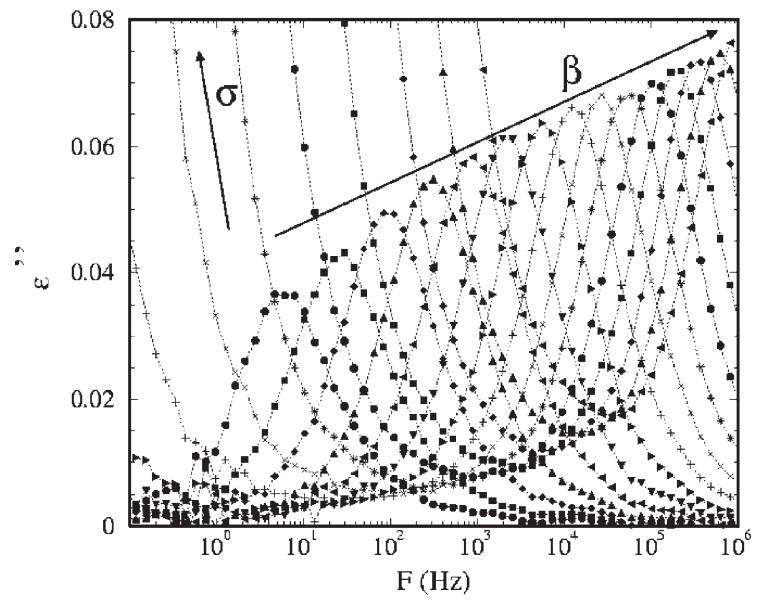

Fig. 1. Frequency and temperature dependence of the dielectric energy loss for the $\beta$ mode of $\left[G_{0}^{\prime}\right]$ (isotherms from -85 to $35^{\circ} \mathrm{C}$ ). 
Table 1

$\mathrm{HN}$ parameters $\left(\alpha_{\mathrm{HN}}\right.$ and $\left.\beta_{\mathrm{HN}}\right)$ evolution with temperature for generation $g=0$ and 2 (extracted from a series of isotherms from $T=-150$ to $50{ }^{\circ} \mathrm{C}$ )

\begin{tabular}{lll}
\hline HN parameters & $g=0\left[G_{0}^{\prime}\right]$ & $g=2\left[G_{2}^{\prime}\right]$ \\
\hline$\alpha_{\mathrm{HN}}$ & $0.90-0.971$ & $0.28-0.41$ \\
$\beta_{\mathrm{HN}}$ & $0.912-1.0$ & 1.0 \\
\hline
\end{tabular}

Evolution of numerical values of $\mathrm{HN}$ parameters of $\beta$ modes as a function of generation are reported in Table 1, for the series of isotherms. Contribution of electrical conductivity $\sigma$, since the isotherm $T=-25{ }^{\circ} \mathrm{C}$, appears on the low frequency tail of $\varepsilon_{\mathrm{T}}^{\prime \prime}(\omega)$; but it does not modify HN fitting. Details of dielectric data analysis were published elsewhere [5]. By TSC, depolarization current exhibits a narrow (quasi non-distributed) $\beta$ relaxation mode. For $\left[G_{2}^{\prime}\right]$, two relaxation regimes are revealed by TSC: the $\alpha$ relaxation mode observed at higher temperature is due to the dielectric manifestation of the glass transition; at lower temperature, the $\beta$ mode due to localized molecular mobility is characterized by relaxation times with an Arrhenius-Eyring like temperature dependence

$\tau(T)=\left(h / k_{\mathrm{B}} T\right) \exp (-\Delta S / R) \exp (\Delta H / R T)$,

where $k_{\mathrm{B}}, h$ and $R$ are Boltzmann's, Planck's and the gas constants, respectively, $\Delta H$ the activation enthalpy and $\Delta S$ the activation entropy. Evolution of depolarization current shape as a function of generation, is shown on Fig. 2. The low tempera-

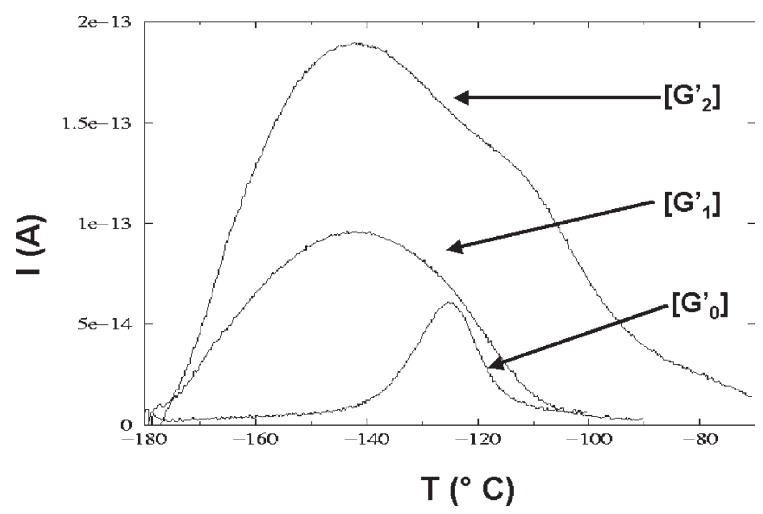

Fig. 2. Complex TSC spectra of $\left[G_{0}^{\prime}\right],\left[G_{1}^{\prime}\right]$ and $\left[G_{2}^{\prime}\right](E=400$ $\mathrm{kV} / \mathrm{m})$. ture mode $\beta_{2}^{\left[G^{\prime}\right]}$ occurs in the same temperature range than $\beta_{0}^{\left[G^{\prime}\right]}$, but its half-width increases. Considering the mobility and the polarity of the aldehyde groups, they can be responsible for the $\beta$ relaxation modes. A detail comparative study [6] of dendrimers of generation 0 , with $\left[G_{0}^{\prime}(\mathrm{P}=\mathrm{O})\right]$ and without aldehyde end groups $\left[G_{0}(\mathrm{P}=\mathrm{O})\right]$ allows us to attribute the low temperature mode to reorientations of aldehyde end groups. Moreover, the substitution of a $\mathrm{P}=\mathrm{S}$ core by a $\mathrm{P}=\mathrm{O}$ core [6], shows that there is a significant interaction with aldehyde group. Dendrimers are good models for analyzing the molecular origin of $\beta$ relaxation modes observed in complex systems. $\beta$ modes are strongly influenced by the complexity (generation). A comparative study of molecules allows us to attribute the low temperature relaxation mode to reorientations of aldehyde end groups. This is a quasi-Debye mode characteristic of a localized molecular mobility. Then, chemical modification of the core reflects the strong interaction of the core with the end groups. When generation increases, a broadening of the $\beta$ relaxation mode is observed. The activation enthalpy evolution of these modes $\left(\beta_{0}^{\left[G^{\prime}\right]}, \beta_{1}^{\left[G^{\prime}\right]}, \beta_{2}^{\left[G^{\prime}\right]}\right)$ is studied. Activation enthalpies of isolated processes are extracted from FP protocol. Activation enthalpies follow the Starkweather's 'line' corresponding to the theoretical activation enthalpy $\Delta H_{0}$ associated with null activation entropy. It obeys the following law [7]

$\Delta H_{0}=R T \ln \left(k_{\mathrm{B}} T / 2 \pi h f_{\mathrm{eq}}\right)$,

where $f_{\text {eq }}$ is the equivalent frequency of TSC ( $f_{\mathrm{eq}} \approx 5 \times 10^{-2} \mathrm{~Hz}$ with our experimental conditions). So secondary relaxations in dendrimers have been associated with non-cooperative mobility of localized dipoles: aldehyde end-groups, in agreement with Starkweather's cooperativity criterion [7]. The combined TSC-DDS analyses will now be applied on more delocalized motions around the glass transition.

\subsection{Glass transition region: main relaxation in biopolymers}

Elastin is an amorphous macromolecule that confers elasticity to tissues such as skin, lung and 
aorta. Containing one third glycine amino acids, elastin is a three-dimensional network with $60-70$ amino acids between two crosslinking points. This complex molecular architecture is important for its elastic properties, insolubility and resistance to several proteases [8]. Since proteins are made from polar repeating units $(\mathrm{CO}-\mathrm{CR}-\mathrm{NH})$, dielectric techniques can be used to develop a relaxation map of elastin around the glass transition temperature determined by DSC at $200{ }^{\circ} \mathrm{C}$. The $\varepsilon^{\prime \prime}$ spectra obtained by DDS and associated with dielectric loss in dry elastin [9] show a great increase at low frequency with rising temperature, due to an important phenomenon of conductivity, that partially hides the $\alpha$ relaxation process corresponding to the dielectric glass transition. When using the $M^{*}$ formalism $\left(M^{*}=1 / \varepsilon^{*}\right)$ the $\alpha$ relaxation is resolved and obeys a Vogel-TammannFulcher (VTF) law, with $T_{\infty}=150{ }^{\circ} \mathrm{C}$ i.e. $\mathrm{T}_{\mathrm{g}}-50^{\circ} \mathrm{C}$ as widely observed for synthetic polymers. Nevertheless, the dielectric relaxation of elastin is close to the degradation zone (around $230{ }^{\circ} \mathrm{C},[10]$ ). On the contrary, the use of TSC spectrometry allows lowering the analysis frequency domain so that the relaxation is shifted towards low temperature, and observed from the glassy state. The complex TSC spectra of freeze-dried elastin recorded after a 400 $\mathrm{kV} / \mathrm{m}$ polarization at 170 and $190{ }^{\circ} \mathrm{C}$ are reported in Fig. 3. A single $\alpha$ relaxation mode, well-defined

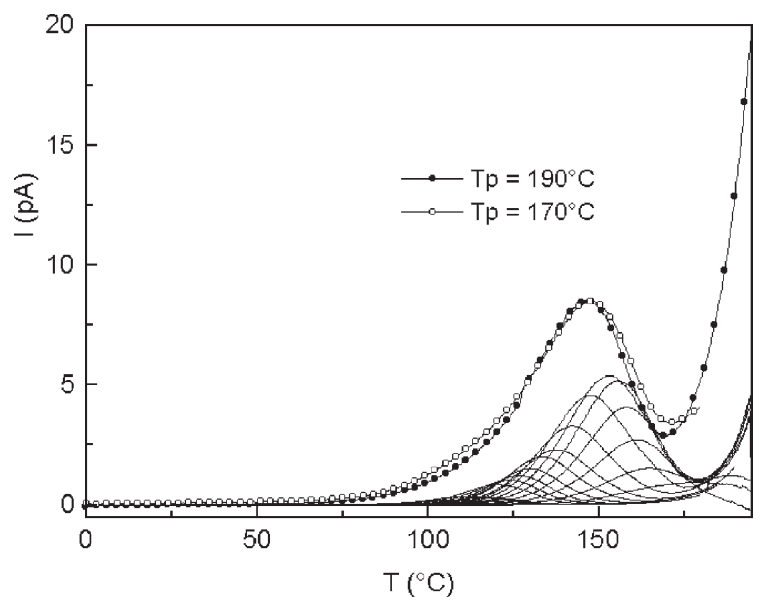

Fig. 3. Complex (line with open and close circle) and elementary (in full line) TSC spectra of freeze-dried elastin. and reproducible with different polarization temperatures and different samples, is observed in the $100-160{ }^{\circ} \mathrm{C}$ temperature range. The following mean characteristic values are $T_{\max }=145^{\circ} \mathrm{C}$ (error bar $4{ }^{\circ} \mathrm{C}$ ) and $I_{\max }=8.8 \times 10^{-12} \mathrm{~A}$ (error bar $\left.1.4 \times 10^{-12} \mathrm{~A}\right)$. As observed in synthetic polymers, the main $\alpha$ relaxation mode of elastin is about one hundred times more intense than the secondary $\beta$ relaxation process observed at $-100{ }^{\circ} \mathrm{C}$ [11]. From $180{ }^{\circ} \mathrm{C}$, a large increase of the depolarization current is noted, corresponding to a conductivity phenomenon, independent from the relaxation process. The FP method is applied to reach the experimental distribution of relaxing species. The set of Debye spectra recorded by shifting the polarization window in the $\alpha$ relaxation zone are reported on Fig. 3. We notice that the temperature dependence of all the relaxation times isolated by the FP method checks an Arrhenius-Eyring law. This observation is in good agreement with the model developed by Eyring [12] and Fröhlich [13] for the description of relaxations in polymers below the glass transition. Fig. 4 shows the variation of the activation enthalpy of elastin as a function of temperature (black circles). To discriminate between cooperative processes, the Starkweather's function (continuous line) has been superimposed on the figure. It can be observed from Fig. 4 that the lowest temperature processes of the main $\alpha$ relaxation obey the null entropy prediction: they reflect the movement of small molecular segments

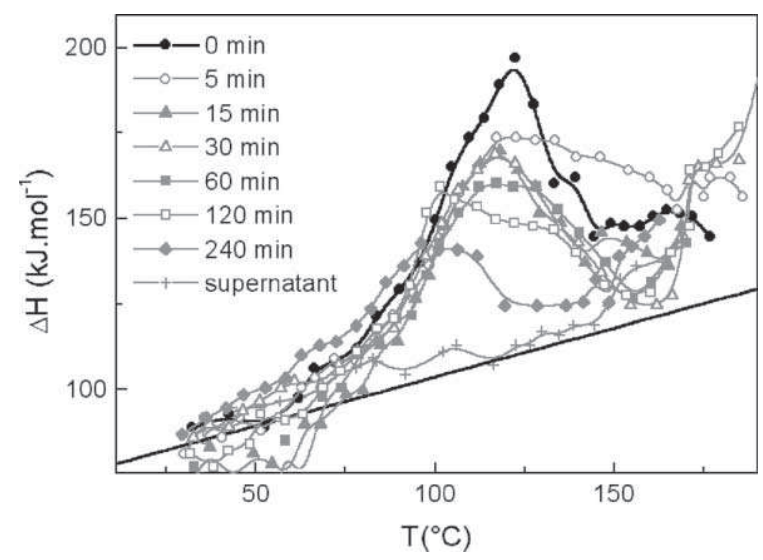

Fig. 4. Activation enthalpy of the elementary relaxation times of different elastins computed from the FP method. 
involving no modification of the environment. A sharp increase of $\Delta H$ is observed above $60-70{ }^{\circ} \mathrm{C}$, and a maximum value of $\Delta H=197 \mathrm{~kJ} / \mathrm{mol}$ is found for $T_{\max }=122.5{ }^{\circ} \mathrm{C}$. So the $\mathrm{FP}$ method allows to scan elementary relaxation processes described by increasing activation entropy and enthalpy and so corresponding to cooperative motions. This behavior that reflects the molecular mobility of dipolar species preceding the glass transition phenomenon has been observed for numerous polymers. To get insight into the physical meaning of $\Delta H$, elastin is gradually hydrolyzed by a specific elastase (porcine pancreatic elastase). The activation enthalpies extracted from the analyses of the differently degraded elastin networks are superimposed on Fig. 4, with the one corresponding to the soluble supernatant (gray curves). $\Delta H_{\max }$ decreases as hydrolysis increases, confirming the WilliamHoffman-Passaglia model [14]: $\Delta H$ can be correlated to the length of the relaxing unit. As for $\Delta H_{\max }$, it is an estimation of the greater scanned dipolar motion in the glassy state associated with the dielectric manifestation of glass transition. To complete our understanding on cooperativity phenomenon, let us consider now the study of delocalized motions on a series of amorphous linear model polymers with various side-length chains.

\subsection{From glass transition to upper region: relax- ations in poly(n-alkyl methacrylates)}

The dielectric study of poly(n-alkyl methacrylates) series (PnAMA), especially the PMMA, the PEMA, the PnBMA and the PnHMA, allows us to determine the influence of chain architecture on relaxations at and above $T_{\mathrm{g}}$, a detailed review of results will be published elsewhere. The study by TSC of samples of PMMA, PEMA, PnBMA and PnHMA in the form of film shows the existence of three dipolar relaxation modes around the glass transition: a $\beta$ secondary mode associated with the flip motion of the ester group around the $\mathrm{C}-\mathrm{C}$ bond which links it to the main chain [15], an $\alpha$ mode ascribed to the dielectric manifestation of the glass transition and an $\alpha^{\prime}$ mode, above $T_{\mathrm{g}}$ [16]. Whereas the $\beta$ mode is weakly influenced by the length of the ester group, the $\alpha$ and $\alpha^{\prime}$ modes are shifted towards lower temperatures when it in- creases. This phenomenon is well known as internal plasticization. The fine structure of these two modes is defined by using the FP method. The relaxation times extracted from elementary spectra isolated in this temperature range for PnBMA, as an example, are reported on Fig. 5 [16]. For all PnAMA studied, the $\alpha$ mode is widely distributed and the relaxation times obey an ArrheniusEyring equation, like elastin. A compensation law [17] is found for PEMA and PnBMA, which highlights the cooperativity of this mode. For PMMA, the compensation phenomenon is not detected, due to the proximity of $\alpha^{\prime}$ mode, contrarily to previous Sauer's observation [18]. For PnHMA, $\beta$ mode interferes with $\alpha$ mode. That cooperative aspect of the $\alpha$ mode is in agreement with Starkweather's interpretation [7] as the activation enthalpies depart from the Starkweather null activation entropy up to the mode maximum temperature. As the activation enthalpy at the mode maximum decreases when the ester group increases, it is influenced by the chain architecture.

As we can see on Fig. 5, relaxation times isolated for the $\alpha^{\prime}$ mode are narrowly distributed, contrary to the $\alpha$ mode. Their temperature dependence is function of chain architecture: for PMMA and PEMA, relaxation times obey an Arrhenius equation whereas a VTF law better describes it for PnBMA and PnHMA. In order to follow the evolution of the modes with frequency, we realize DDS experiments for each sample, with and without a blocking PTFE electrode. Relaxation

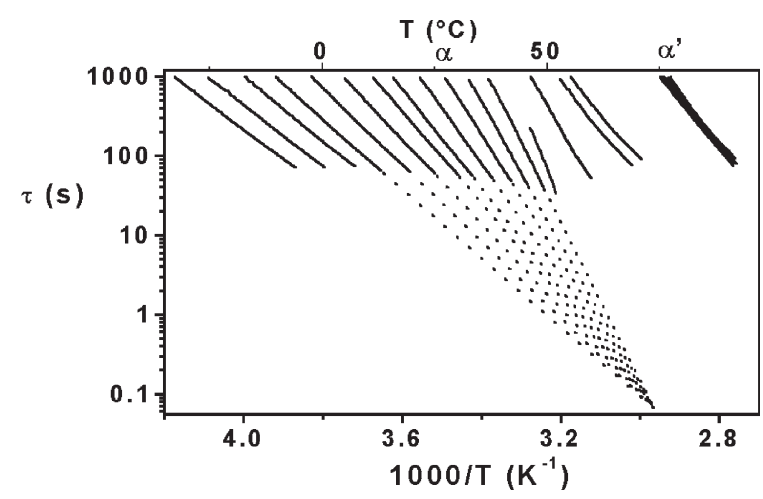

Fig. 5. Variation of relaxation times extracted from elementary spectra isolated by FPs in the case of PnBMA. 
times are extracted from spectra by using $\mathrm{HN}$ fit. Those of PnBMA associated with PTFE, are reported on Fig. 6 [19]. An interesting fact with $\operatorname{poly}(n$-alkyl methacrylates) is that the well-known merging of the $\alpha$ and $\beta$ modes is accessible in the DDS window [20]. Indeed, we can see on Fig. 6 that, when frequency increases, the $\alpha$ and $\beta$ modes come closer together and, at high frequency, only one mode, called $(\alpha \beta)$, subsists. As pointed out by Garwe et al. [21], for all the samples of the series, we can find an onset point of the $\alpha$ mode: when frequency increases, the dielectric relaxation strength decreases and the $\alpha$ mode vanishes before it reaches the $(\alpha \beta)$ mode. Whereas the $\beta$ mode is weakly influenced by the chain architecture, the $\alpha$ and $(\alpha \beta)$ modes are shifted towards lower temperatures when the ester group increases. The merging point, or more precisely, the $\alpha$ onset point is shifted towards lower frequencies and temperatures with increasing size of the alkyl side chain. We note that the relaxation time of the $\alpha$ mode obeys a VTF equation and we observe, as in elastin [9], a crossover from a VTF behavior by DDS to an Arrhenius one by TSC, witness of a crossover from an equilibrium state in DDS to a non-equilibrium one below $T_{\mathrm{g}}$ in TSC. At low frequencies and high temperatures, conductivity appears. In order to remove it, we realize experiments with a blocking

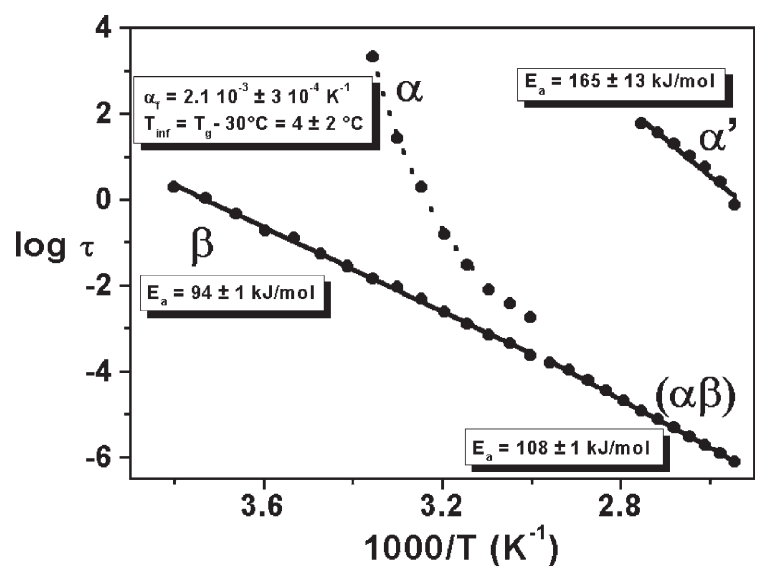

Fig. 6. Variation with frequency and temperature of relaxations times extracted from DDS spectra for PnBMA/PTFE. Straight lines represent the Arrhenius fit and dashed line the VTF fit. electrode. It does not influence the results obtained for the $\beta, \alpha$ and $(\alpha \beta)$ modes, but an additional relaxation phenomenon, $\alpha^{\prime}$, occurs instead of conductivity. This dielectric relaxation is shifted towards lower temperatures when the ester group increases. Up to now, we still do not know the origin of this mode but we can relate it directly (dipolar) or indirectly (ionic) to a motion of macromolecular chains. It should be noted that this mode fits in temperature and frequency with the $\alpha^{\prime}$ dipolar relaxation mode found by TSC.

\section{Discussion}

From these studies carried out on different materials - fractal architecture macromolecules (dendrimers), crosslinked biological macromolecules (elastin) and linear polymer (poly- $n$-alkyl methacrylates) - the combination of TSC and DDS analyses is shown to be particularly suitable to achieve a better knowledge about the molecular dynamics of complex systems. This fact is based firstly on the interesting enlargement of the analysis frequency range in the low frequency side by using TSC experiment as a complementary technique of DDS and secondly on the accuracy of the TSC technique to determine the fine structure of complex relaxation modes. Results obtained from these studies confirm the global scheme of dynamics specific of glass forming materials reported on the Fig. 7. This figure displays elementary dynamics involved in each complex relaxation processes $\left(\beta, \alpha\right.$ and $\left.\alpha^{\prime}\right)$ as can be extracted experimentally from the TSC-FP method and numerically from analysis of the relaxation times distribution determined by DDS through regularization method [22,23]. This scheme indicates the continuity of TSC and DDS results concerning the secondary $\beta$ relaxation dynamics [23]. The $\beta$ relaxation mode, associated with localized molecular motions, involves elementary dynamics described, in the whole temperature domain, by an Arrhenius temperature dependence. The weak enthalpy distribution observed between elementary dynamics may be related to the narrow local density distribution at these temperatures. The dielectric techniques allow to probe successively all species 


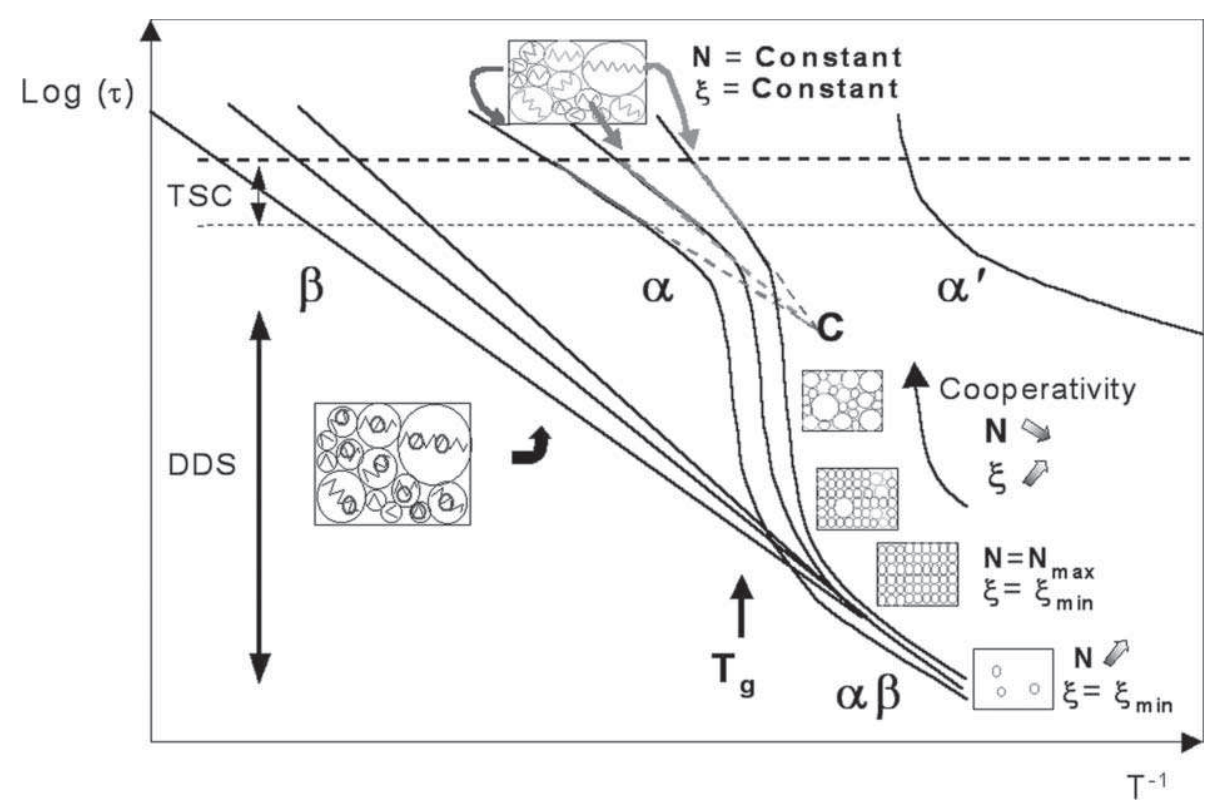

Fig. 7. Arrhenius representation scheme of glass forming materials dynamics in the temperature range of $\beta$, $\alpha$, and $\alpha^{\prime}$ relaxation modes ( $N$ is the number and $\xi$ the mean size of CRR in the system).

localised in more and more constraining environments. The activation entropy close to zero for all $\beta$ elementary relaxation processes means that no evolution of the physical structure of local environments is expected during the movements of involved low size relaxing units. Concerning the $\alpha$ relaxation, the involved elementary relaxation processes exhibit a crossover between their VFT and their Arrhenius behaviour law observed above and below the glass transition temperature by DDS and TSC respectively. For the study of $\alpha$ relaxation process, associated with delocalised molecular motions, TSC and DDS are complementary experiments. In the Adam and Gibbs model extended to take into account the $\alpha$ and $\beta$ relaxation merging [15,21], at temperatures largely higher than $T_{\mathrm{g}}$ the system is composed on an increasing number of elementary cooperative rearrangement regions (CRR) when the temperature is decreased. The bifurcation between $\alpha$ and $\beta$ relaxation occurs when the system is entirely filled by such elementary CRR. Decreasing the temperature from this point, the cooperativity of relaxing species related to the density of material increases dramatically. The size of CRR increases and at the same time their number decreases: there is a coalescence phenomenon. The distribution of relaxation times observed in this temperature range $\left(T>T_{\mathrm{g}}\right)$ is associated with the size distribution of CRR [23]. The VFT temperature dependence of relaxation times may be related to the dynamics of relaxing species having a temperature dependent length. At $T_{\mathrm{g}}$, the system is frozen. The size and the number of CRR become temperature independent for $T<T_{\mathrm{g}}$. By TSC, all size distributed CRR are successively probed and all elementary relaxation times determined by TSC-FP exhibiting an Arrhenius temperature dependence are representative of dynamics of relaxing species with constant length. The more the size of the relaxing specie is important, the more the motion of this species is constrained by its local environment: the activation enthalpy of its dynamics increases. However, in return, the environment is particularly perturbed ( $\Delta S$ increases) when the motion has a large amplitude [24]. The activation entropy is so related to the activation enthalpy traducing the compensation phenomenon. As for the $\alpha^{\prime}$ relaxation process, although several works are still scheduled, it has been assignated to the disentanglement of 
macromolecular chains, allowing the mobility to delocalize along entire chains. This mode is characteristic of amorphous phase heterogeneity in glass transition upper region.

\section{Conclusion}

The combination of DDS and TSC allows us to propose a phenomenological model explaining dielectric complexity (heterogeneity) of amorphous polymers. Owing to the broad temperature and frequency range, it becomes possible to follow the evolution of metastable amorphous phases and to propose a structural interpretation. Finally, by using model samples, the understanding of the molecular origin of dielectric properties may be greatly improved.

\section{References}

[1] C. Bucci, R. Fieschi, Phys. Rev. Lett. 12 (1964) 16.

[2] M.L. Lartigue, B. Donnadieu, G. Galliot, A.M. Caminade, J.P. Majoral, J.P. Fayet, Macromolecules 30 (1997) 7335.

[3] J.P. Majoral, A.M. Caminade, in: Topics in Current Chemistry, vol. 197, Springer, Berlin, 1998, p. 79.

[4] S.A. Hurve, D. Appelhans, J. Prigann, B.I. Voit, F. Kremer, Macromolecules 33 (2000) 3762.

[5] E. Dantras, C. Lacabanne, A.M. Caminade, J.P. Majoral, Macromolecules 34 (2001) 3808 .
[6] E. Dantras, A.M. Caminade, J.P. Majoral, C. Lacabanne, J. Phys. D 35 (2002) 5.

[7] H.W. Starkweather, Macromolecules 14 (1981) 1277.

[8] T.F. Linsenmayer, in: E.D. Hay (Ed.), Cell Biology of Extracellular Matrix, 2nd Ed., Plenum, New York, 1991, p. 4.

[9] V. Samouillan, A. Lamure, C. Lacabanne, Chem. Phys. 255 (2000) 255.

[10] V. Samouillan, J. Dandurand-Lods, A. Lamure, E. Maurel, C. Lacabanne, G. Gerosa, A. Venturini, M. Spina, J. Biomed. Mater. Res. 46 (1999) 531.

[11] V. Samouillan,, A. Lamure, E. Maurel, J. Dandurand, C. Lacabanne, M. Spina, J. Biomater. Sci. Polym. Ed. 11 (2000) 583.

[12] H. Eyring, J. Chem. Phys. 4 (1936) 283.

[13] H. Fröhlich, in: Theory of Dielectrics, 2nd Ed., Oxford University, Oxford, 1958.

[14] J.D. Hoffmann, G. Williams, E. Passaglia, J. Polym. Sci 14 (1966) 173.

[15] M. Beiner, F. Garwe, K. Schröter, E. Donth, Colloid. Polym. Sci 272 (1994) 1439.

[16] E. Dudognon, A. Bernès, C. Lacabanne, Macromolecules 34 (2001) 3988.

[17] C. Lacabanne, A. Lamure, G. Teyssèdre, A. Bernès, M. Mourgues, J. Non-Cryst. Solids 172-174 (1994) 884.

[18] B.B. Sauer, P. Avakian, Polymer 33 (1992) 5128.

[19] E. Dudognon, A. Bernés, C. Lacabanne, J. Phys. D 35 (2002) 9.

[20] N.G. McCrum, B.E. Read, G. Williams, Anelastic and Dielectric Effects in Polymeric Solids, John Wiley, New York, 1991.

[21] F. Garwe, A. Schönhals, H. Lockwenz, M. Beiner, K. Schröter, E. Donth, Macromolecules 29 (1996) 247.

[22] J. Menegotto, P. Demont, A. Bernes, C. Lacabanne, J. Polym. Sci.: Part B: Polym. Phys. 37 (1999) 3494.

[23] S. Matsuoka, X. Quan, Macromolecules 24 (1991) 2770.

[24] J.P. Menegotto, Paul Sabatier University thesis, 1999. 